\title{
Efficient Transmitters and Receivers for High-Power Wireless Powering Systems
}

\author{
Zoya Popovic, Tibault Reveyrand, Scott Schafer, Michael Litchfield, Ignacio Ramos, Sean Korhummel \\ Dept. of Electrical, Computer and Energy Engineering \\ University of Colorado \\ Boulder ,U.S.A. \\ zoya@colorado.edu
}

\begin{abstract}
The efficiency of a wireless powering system is maximized when the power transmitter power-added efficiency (PAE), power receiver conversion efficiency $\left(\eta_{C}\right)$ and wireless coupling efficiency $\left(\eta_{w}\right)$ are maximized. This paper focuses on a general approach to the design of an efficient transmitter and receiver of a high-power wireless powering system, which is valid for any type of wireless power coupling. Experimental results for high-efficiency power transmitters and receivers at various frequencies and with various power levels are discussed.
\end{abstract}

Keywords-wireless power transfer, rectifier, amplifier, high efficiency, time-reversal

\section{INTRODUCTION}

Wireless powering systems, whether inductive [1], resonant [2], high-directive beaming [3,4], omni-directional rf flooding [5] or multi-mode guided wave [6] all contain a DC to AC (RF) converter, an electromagnetic wireless power transfer method, and an AC (RF) to DC converter. Higher power levels with near-field reactive electromagnetic coupling are typically used for applications such as automobile powering, while plane waves carrying ultra-low power levels are scavenged or harvested from known transmitters typically in the UHF or microwave range $[7,8]$. High power levels are also used in some beaming applications with plane waves and directive antennas or arrays [9]. Powering of personal devices and implants is usually performed at medium power levels inductively $[10,11]$, though multi-mode cavities have also been proposed [6].

At the receiver end, for low power levels, Schottky diodes have been used almost exclusively for rectifiers integrated with antennas with relatively high demonstrated efficiencies at as low as $1 \mathrm{uW} / \mathrm{cm}^{2}$ incident power levels, e.g. [11]. In the UHF and microwave frequency range, diodes have power limitations, and power rectifiers are made either with distributed rectenna arrays or can be also implemented with transistors, e.g. [12].

In the case of low-power wireless powering, the efficiencies are not critical since the power levels are not expensive to produce and do not result in heating. In this case, the efficiency is maximized at the receiver in order to turn on the rectifying element and enable charging of a storage device [5]. In the high-power case, efficiency is much more relevant, as the power dissipation can be very large. In order to optimize efficiency of the wireless powering system, the individual efficiencies of the transmitter, electromagnetic power transfer and the receiver need to be optimized. Additionally, in the case of near-field power transfer, the receiver and transmitter are coupled and this loading needs to be taken into account.

This paper aims at presenting state-of-the-art results in transmitter and receiver efficiencies at frequencies in the $\mathrm{S}$ and $\mathrm{X}$ bands for power levels greater than $5 \mathrm{~W}$, using GaN device technology. Figure 1 below shows the general system block diagram. The transmitter can be viewed as a DC to RF converter, and although fundamentally this is an oscillator, in reality the transmitter efficiency will be dominated by the power-added efficiency (PAE) of the final stage power amplifier. The output signal can be modulated with some lower frequency, as indicated in the DC path, though it can also be accomplished through the input path. The wireless power transfer is performed electromagnetically and can be represented by an equivalent network which can either couple the transmitter and receiver, or one which isolates them. The power receiver is a power amplifier operated as a rectifier. The DC power at the output is delivered to a resistive load. The rectifier stage can be synchronous, requiring an input signal which reduces overall efficiency, or self-synchronous with no input signal.

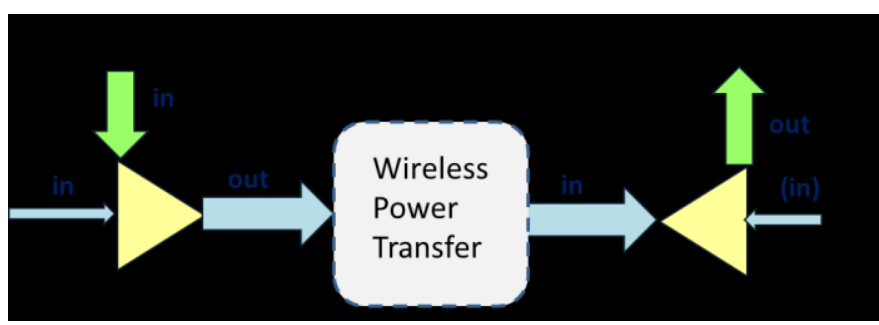

Figure 1. General block diagram of a wireless power transfer system, including the transmitter final stage power amplifier, the wireless power transfer block and the power receiver block. In this paper, high-efficiency power amplifier circuits are used for both the transmitter and the power rectifier. The DC output is delivered to a resistive load, discussed later in the paper.

The paper first discusses efficient power amplifier transmitter design, followed by an investigation of efficient power rectifier design and characterization. A duality between the transmitter and receiver is then presented, concluding with a discussion of future technology trends related to efficient wireless powering system components. 


\section{HIGH-EFFICIENCY POWER AMPLIFIERS}

The efficiency of the transmitter in Fig. 1 is dominated by the PAE of the final stage amplifier. At lower microwave frequencies, hundreds of watts are achievable with low-cost LDMOS devices with efficiencies above $50 \%$, while the best demonstrated efficiencies with $10-100 \mathrm{~W}$ output power levels at S-band have been demonstrated with GaN HEMTs. A good overview of current status of power amplifiers using various technologies can be found in [13]. In this paper, we focus on GaN high efficiency harmonically-terminated PAs for wireless transmitters, which have also been characterized as power rectifiers: (1) an S-band 7-W PA using a 0.25um GaN device and (2) a 3-W X-band PA using a $0.15 \mathrm{um} \mathrm{GaN} \mathrm{device.}$

In [14], a PA based on the Triquint TGF2023-02 HEMT $12-\mathrm{W}$ bare die, is demonstrated to exhibit $7 \mathrm{~W}$ output power and over $84.6 \%$ PAE with a drain voltage of $31 \mathrm{~V}$ and a RF signal at $2.14 \mathrm{GHz}$. The drain matching of the transistor is designed to produce class- $\mathrm{F}^{-1}$ time-domain waveform at the transistor's output reference plane. The gate matching is a resonant circuit at $2.14 \mathrm{GHz}$. This PA is implemented in a hybrid circuit, and was characterized as both a PA and a rectifier, discussed in the next section.

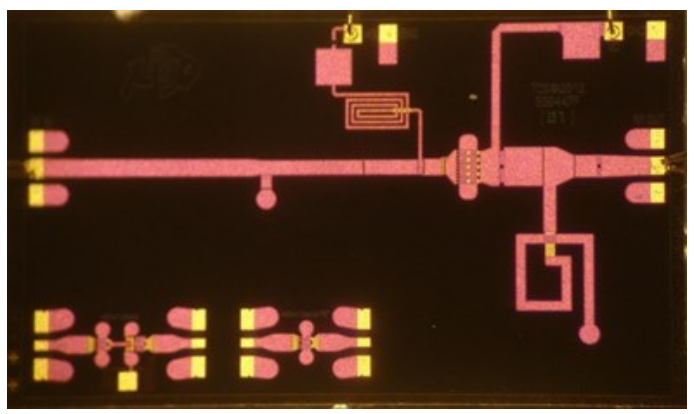

Figure 2. Photograph of a $10-\mathrm{GHz}$ single-stage PA with complex harmonic terminations designed for high efficiency with $3 \mathrm{~W}$ output power. The amplifier itself measures less than $2.5 \times 2.5 \mathrm{~mm}$, though the chip is larger due to reticle dimensions and layout. Test devices are in the lower left corner.

Many researchers have detailed design of various harmonically terminated PA classes, such as $\mathrm{F}, \mathrm{F}^{-1}, \mathrm{E}$, J, etc. and continuous classes of operation. All these classes assume fixed harmonic terminations which are either short or open circuits. A more general discussion is given in [15] for arbitrary complex-valued harmonic terminations, and this method was used in the design of a $10-\mathrm{GHz}$ MMIC PA in the TriQuint 0.15 um GaN on $\mathrm{SiC}$ process. This MMIC is shown in Figure 2 and its performance in Figure 3. The device is biased in Class-B operation in deep pinch-off. The best performance is measured at $10.2 \mathrm{GHz}$ with peak PAE of $69 \%$ with $\mathrm{P}_{\text {OUT }}=2.7 \mathrm{~W}$ and a peak $\mathrm{P}_{\text {OUT }}=3.7 \mathrm{~W}$ with $\mathrm{PAE}=56 \%$. The large signal gain of this $\mathrm{PA}$ is $\mathrm{G}=8 \mathrm{~dB}$, and over a bandwidth of $600 \mathrm{MHz}$ the PAE $>60 \%$. This state-of-the-art MMIC PA is also characterized as a rectifier as discussed in the next section.

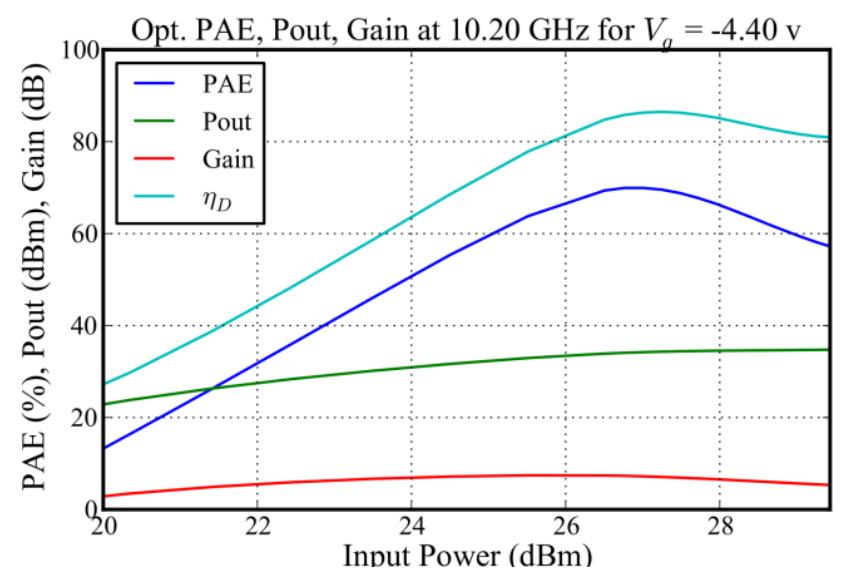

Figure 3. Measured performance of the MMIC GaN PA at $10.2 \mathrm{GHz}$.

\section{High-EFFICIENCY POWER RECTIFIERS}

High-efficiency harmonically terminated rectifiers with diodes and transistors at S-band frequencies were first discussed in [16]. A synchronous power rectifier at UHF frequencies was then demonstrated in [17], and a selfsynchronous rectifier with $>80 \%$ efficiency was discussed in [18], where the $2.14 \mathrm{GHz} \mathrm{PA}$ from the previous section was tested in rectifier mode.

The setup for characterizing the PA as a rectifier is shown in Figure 4. The power is input into the drain, with the drain bias turned off and a resistor load connected to the drain bias Tee. A 4-channel receiver (LSNA) is used to measure the timedomain waveforms at the input and output, and a passive tuner augmented by an active loop provides a wide range of impedances at the gate terminal of the transistor PA. The DC load resistor RD is varied for optimal performance, as is the RF load at the gate. The gate is biased in cutoff. The input power into the drain is of the same order as the output power when the device operates as a PA.

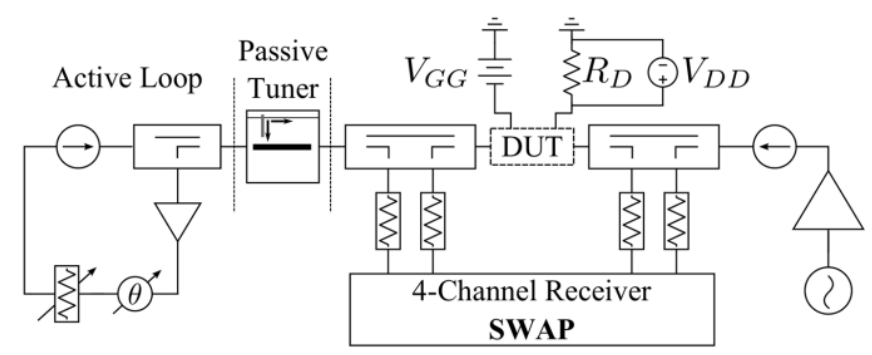

Figure 4. Block diagram of test setup for characterizing PAs as rectifiers using a 4-channel receiver (LSNA, or SWAP). The gate RF impedance and DC resistor at the drain are adjusted for optimal RF to DC conversion efficiency. 
The PA from [14] was tested as a rectifier and demonstrated an output power of $10 \mathrm{~W}$ with $85 \%$ conversion efficiency when operated in self-synchronous mode into a $98-\mathrm{ohm}$ DC load, as detailed in [16].

The PA from Figure 2 was tested as in Figure 4 and its efficiency as a rectifier is shown in Figure 5 as a function of available RF power at the drain input and for a load of 100ohms. The input impedance at the drain was measured as a function of input power and is shown in Figure 6. We can conclude from these results that the PA and rectifier efficiencies are very close, that the rectification efficiency is low for low input power and that the impedance looking into RF drain (rectifier input) varies with input power, which will have an effect on near-field wireless power transfer systems and needs to be carefully characterized.

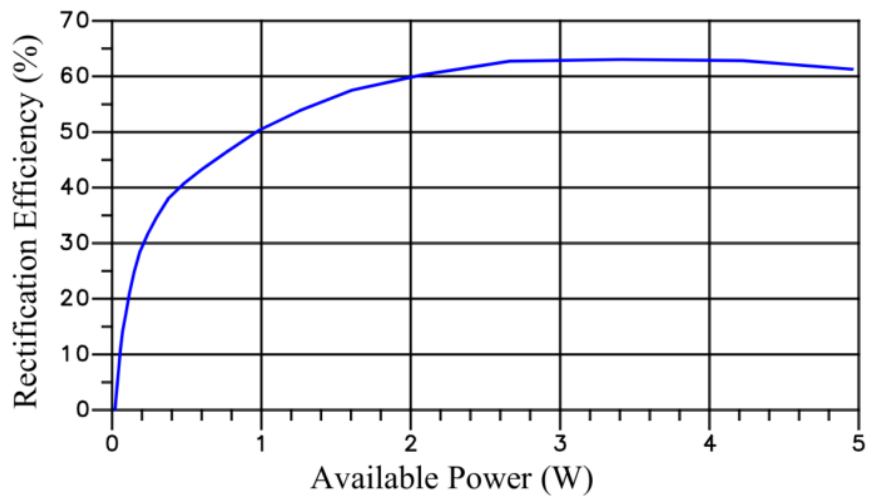

Figure 5. Measured rectification efficiency of the X-band MMIC PA from Figure 2 at $10.2 \mathrm{GHz}$ with a fixed 100 -ohm DC load and an optimal RF load at the gate. The gate is biased in cutoff at $-4 \mathrm{~V}$.

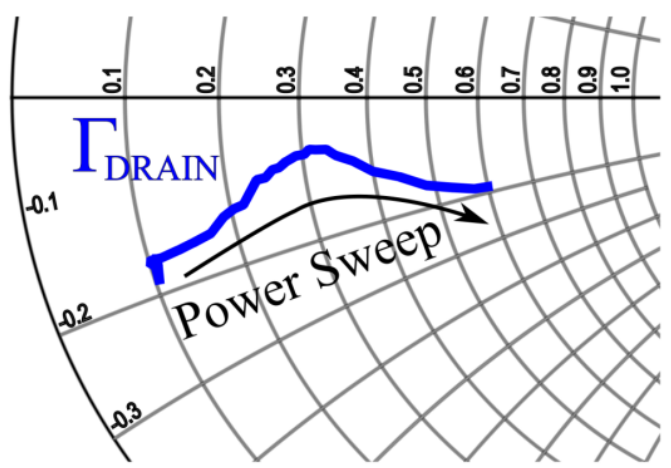

Figure 6. Measured input impedance looking into the drain of the PA operating as a rectifier at $10.2 \mathrm{GHz}$ as a function of input power.

\section{DISCUSSION AND CONCLUSIONS}

In conclusion, it is possible to operate a high-power highefficiency power amplifier as a high-efficiency power rectifier. This property can be theoretically derived under ideal assumptions using Fourier analysis as shown for class $\mathrm{F}^{-1}$ mode case in [16]. This is made possible by the fact that a rectifier and amplifier have inverse currents, sometimes referred to as "time-reversal". It is important to note that this duality is true for any class of operation: if one can design an efficient PA with an output power Pout, it will operate as an efficient rectifier when Pout is input into the drain port.

It would be interesting to show these waveforms in simulations using a nonlinear transistor model. Unfortunately, most transistor models do not adequately describe the performance for negative drain currents in the $3^{\text {rd }}$ quadrant of the IV characteristics. Such a model can however be found in [19], so simulations were performed with this $8 \times 75$ m GaN HEMT model. This non-linear model includes : non-linear capacitances $\mathrm{Cgs}, \mathrm{Cgd}$ and $\mathrm{Cds}$; gate-souce and gate-drain diodes ; breakdown and trapping effects ; and reproduces the I-V characteristics for positive and negative values of the drain voltage. The resulting waveforms for the amplifier and rectifier for a class-F design are shown in Figure 7 and demonstrate in simulation the PA-rectifier duality.
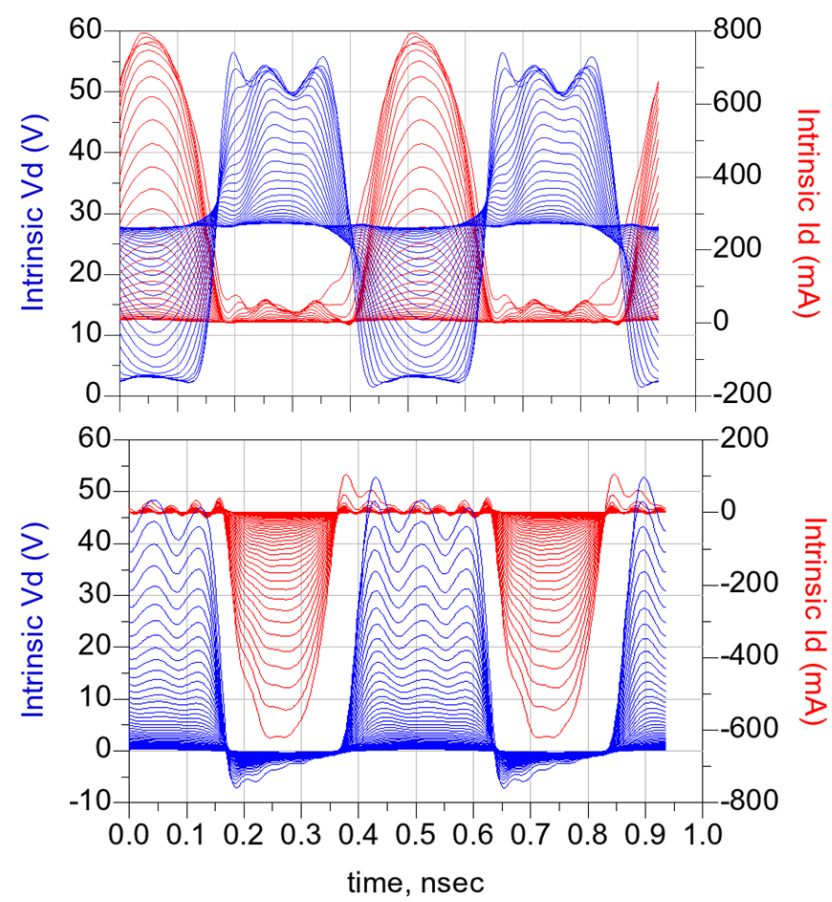

Figure 7. Simulated time-domain waveforms at the virtual drain of a class-F PA (top) and same PA operating as a self-synchronous rectifier (bottom) show dual operation with inverse current but the same shape of waveforms, which will result in the same high efficiency due to the short overlap between the voltage and current. The simulations are done at $2 \mathrm{GHz}$ in this case. 
Some important conclusions relevant to wireless powering systems can be drawn from the results presented in this paper as follows:

- High-efficiency PA design techniques can be used to design both an efficient transmitter and efficient receiver for moderate and high-power wireless powering systems;

- For high-coupling coefficient near-field reactive power transfer, the same PA circuit can be used as a rectifier, and the rectifier-PA loading needs to be taken into account in circuit design;

- For far-field beaming, the power at the receive end needs to be calculated and a PA scaled in power compared to that at the transmit end is the most likely resulting requirement;

- Although the results in this paper were focused on an X-band MMIC GaN implementation, the methodology is general and applies to any frequency, any device technology and any type of circuit implementation and electromagnetic coupling between transmitter and receiver.

In the final version of the paper, the experimental efficiency of a complete wireless powering system based on the X-band MMIC PA from Fig.2 will be discussed.

\section{ACKNOWLEDGMENT}

This work was funded in part by ONR through the DARPA Micro-power conversion (MPC) program and in part by a Hudson Moore Jr. Endowed Chair at the University of Colorado, Boulder. The authors are grateful to Prof. Dragan Maksimovic for many helpful discussions.

\section{REFERENCES}

[1] Covic, G.A.; Boys, J.T., "Inductive Power Transfer," Proceedings of the IEEE, vol.101, no.6, pp.1276,1289, June 2013.

[2] A. Kurs, A. Karalis, R. Moffatt, J. D. Joannopoulos, P. Fisher, M Soljacic, "Wireless power transfer via strongly coupled magnetic resonances.," Science, vol. 317, no. 5834, pp. 83-86, 2007.

[3] Shinohara, H. Matsumoto, "Experimental study of large rectenna array for microwave energy transmission," IEEE Trans. Microwave Theory Techn., vol. 46, pp. 261 -268, Mar. 1998.
[4] W.C. Brown, "The history of power transmission by radio waves," IEEE Trans.Microw.Theory Techn., vol. 32, pp. 1230-1242, Sept. 1984. (Also: http://www.youtube.com/watch?v=tlv9WV7rjbM)

[5] Z. Popovic, E. Falkenstein, D. Constinett, R. Zane, "Low-power far-field wireless powering for wireless sensors," Proceedings of the IEEE, Special Issue on Wireless Powering, Vol.101, No. 6, pp. 1397 - 1409, June 2013.

[6] Rahimizadeh, S.; Korhummel, S.; Kaslon, B.; Popovic, Z., "Scalable adaptive wireless powering of multiple electronic devices in an overmoded cavity," Wireless Power Transfer (WPT), 2013 IEEE, vol., no., pp.84,87, 15-16 May 2013

[7] M. Piñuela, P. D. Mitcheson, Stepan Lucyszyn, "Ambient RF Energy Harvesting in Urban and Semi-Urban Environments," IEEE Trans. Microwave Theory Techn,. vol.61, no.6, pp. 2715-2726, June 2013

[8] R. Vias, H. Nishimoto, M. Tentzeris, Y. Kawahara, T. Asami, "A Battery-Less, Energy Harvesting Device for Long Range Scavenging of Wireless Power from Terrestrial TV Broadcasts," IEEE 2012 IMS Digest, Montreal, Canada, June 2012.

[9] R. Dickinson, "Performance of a high-power, 2.388-GHz receiving array in wireless power transmission over 1.54 km," Microwave Symp. Digest, IEEE-MTT-S Intern., pp. 139 -141, June 1976

[10] J.S. Ho, S. Kim, A.S.Y. Poon, "Midfield wireless powering for implantable systems," Proc. IEEE, 101, June 2013.

[11] J.A. Hagerty, F. Helmbrecht, W. McCalpin, R. Zane, Z. Popovic, "Recycling ambient microwave energy with broadband antenna arrays," IEEE Trans. Microwave Theory Techn., vol.52, no.3, pp. 1014-1024, March 2004.

[12] M. Roberg, T. Reveyrand, I. Ramos, E.A. Falkenstein, Z. Popović, "High-Efficiency Harmonically Terminated Diode and Transistor Rectifiers," IEEE Trans. Microwave Theory Tecnh., Vol. 60, No.12, pp.4043-4052, Dec. 2012.

[13] Special Issue on Power Amplifiers, IEEE Trans. Microwave Theory Techn., Vol. 60, No. 6., June 2012

[14] Roberg, M., Hoversten, J., and Popovi'c, Z.: 'GaN HEMT PA with over $84 \%$ power added efficiency', Electronics Letters, 2010, 46, (23), pp. 1553-1554.

[15] "Analysis of High Efficiency Power Amplifiers with Arbitrary Output Harmonic Terminations" M. Roberg, Z. Popovic, IEEE Trans. Microwave Theory Techn., pp. 2037-2048, Aug. 2011.

[16] M. Roberg, T. Reveyrand, I. Ramos, E.A. Falkenstein, Z. Popović, "High-Efficiency Harmonically Terminated Diode and Transistor Rectifiers," IEEE Trans. Microwave Theory Tecnh., Vol. 60, No.12, pp.4043-4052, Dec. 2012.

[17] J. A. Garc'1a, R. Marante, M. N. Ruiz, and G. Hern'andez, "A 1GHz Frequency-Controlled Class E2 DC/DC Converter for Efficiently Handling Wideband Signal Envelopes," pp. 2-5, 2013.

[18] T. Reveyrand, I. Ramos, Z. Popovic, "Time-reversal duality of highefficiency RF power amplifiers," IET Electronics Lett, Vol.48, No.25, Dec. 6, 2012

[19] Callet, G., Faraj, J., Jardel, O., Charbonniaud, C., Jacquet, J.C., Reveyrand, T., Morvan, E., Piotrowicz, S., Teyssier, J.P. and Quéré R.:'A new nonlinear HEMT model for AlGaN/GaN switch applications', International Journal of Microwave and Wireless Technologies, 2010, 2, (3-4), pp. 283-291 